\title{
Moments of the latitudinal dependence of the sunspot cycle: a new diagnostic of dynamo models
}

\author{
S. K. Solanki ${ }^{1}$, T. Wenzler ${ }^{1,2}$, and D. Schmitt ${ }^{1}$ \\ 1 Max-Planck-Institut für Sonnensystemforschung, 37191 Katlenburg-Lindau, Germany \\ e-mail: [solanki; schmitt] @mps.mpg.de \\ 2 Institute of Astronomy, ETH Zentrum, 8092 Zurich, Switzerland \\ e-mail: wenzler@astro.phys.ethz.ch
}

Received 30 September 2005 / Accepted 6 March 2008

\section{ABSTRACT}

\begin{abstract}
Aims. The latitude-distribution of solar activity as represented by sunspots is studied.
Methods. We first determined the latitudinal distribution of a sunspot cycle by integrating the butterfly diagram at each latitude over the length of each cycle. We then formed the five lowest moments of the latitudinal distribution of all complete sunspot cycles since 1874 and compared these moments with each other.

Results. The three lowest moments correlate remarkably well with each other. For example, the mean latitude of the sunspots during a cycle and the latitude range are correlated at the 0.96 level. A clear asymmetry is seen between the two hemispheres, with the southern solar hemisphere showing consistently stronger and more positive correlations than the northern hemisphere. When applied to different simple dynamo models, the same analysis reveals significant differences between the models and demonstrates that such moments are a useful diagnostic in distinguishing between dynamo models. Remarkably, dynamos without a meridional flow provide results closer to those of the Sun's northern hemisphere, while a dynamo with a meridional flow produces fields more like those in the Sun's southern hemisphere. This may provide a clue to the cause of the well-known north-south asymmetry of solar activity.
\end{abstract}

Key words. Sun: activity - methods: data analysis - Sun: sunspots - Sun: magnetic fields

\section{Introduction}

The cyclic behaviour of solar magnetic activity and its cause, the Sun's dynamo, are widely studied facets of the Sun (see the volumes edited by Harvey 1992; Wilson 2002; Ferriz-Mas \& Núñez 2003; and the reviews by Ossendrijver 2003; Charbonneau 2005). Nonetheless, the origin of the Sun's cyclic behaviour is not fully understood. One aspect which complicates the study and analysis of the solar cycle is that the activity cycle is not a periodic phenomenon, but rather every cycle is different from all others, with its own amplitude, length and shape. There have been numerous attempts to find relations between the different parameters of a cycle in order to constrain various dynamo models of magnetic field generation. Particularly well known is the strong relation found by Waldmeier (1935) between the amplitude of a cycle and the length of its rising phase (with weaker relations existing for the length of the descending phase and the whole cycle; cf. Hathaway et al. 2002). Relations have also been found between the amplitude of a given cycle and the length of an earlier cycle (e.g. Solanki et al. 2002). Differences in the amplitudes of even and odd sunspot cycles (Gnevyshev \& Ohl 1948; Wilson 1988; Mursula et al. 2001) and the asymmetry of the rise to maximum and fall to minimum (Hathaway et al. 1994) are other interesting properties of the sunspot cycle. An overview of sunspot cycle properties has been given by Usoskin \& Mursula (2003), while the cycle properties deduced from Zürich sunspot number (Waldmeier 1961) and group sunspot number (Hoyt \& Schatten 1998) records have been compared by Hathaway et al. (2002).
All of these investigators adopted sunspots as proxies of solar activity, which is reasonable, given that sunspot observations provide the longest running direct records of solar activity (Wolf 1852; Waldmeier 1961). They considered the cycle as a time series and in general neglected the latitudinal distribution of solar activity (for exceptions see, e.g. Antalova \& Gnevyshev 1983; Pelt et al. 2000).

Here we took the opposite approach and considered only the latitudinal dependence of sunspot areas within a cycle. Sunspot latitudes have been regularly and reliably measured for less than 2 centuries, so that this restricts the length of time over which they can be analysed. Since 1874, measurements of sunspot areas are also available, which have the advantage that they are proportional to the total magnetic flux contained in the sunspots (Solanki \& Schmidt 1993). In order to be independent of evolution effects within a cycle we integrated over the life time of a cycle and thereby distilled the latitudinal dependence of the sunspot area. We then computed the moments of each such distribution and searched for relations between them.

In addition to solar data we also investigated the output of a set of simple dynamo models in order to judge the diagnostic capabilities of the relations between moments of the Sun's activity cycle. The motivation for this work was to develop a new diagnostic of the solar cycle and of models of the generation of the SunŠs magnetic field. Therefore, the dynamo models used here are not meant to give a realistic representation of the real Sun, but rather to test the extent to which the diagnostic found in the observational data can distinguish between dynamo models with different ingredients. As causes of variability of the solar cycle we considered stochastic fluctuations of the $\alpha$-effect 


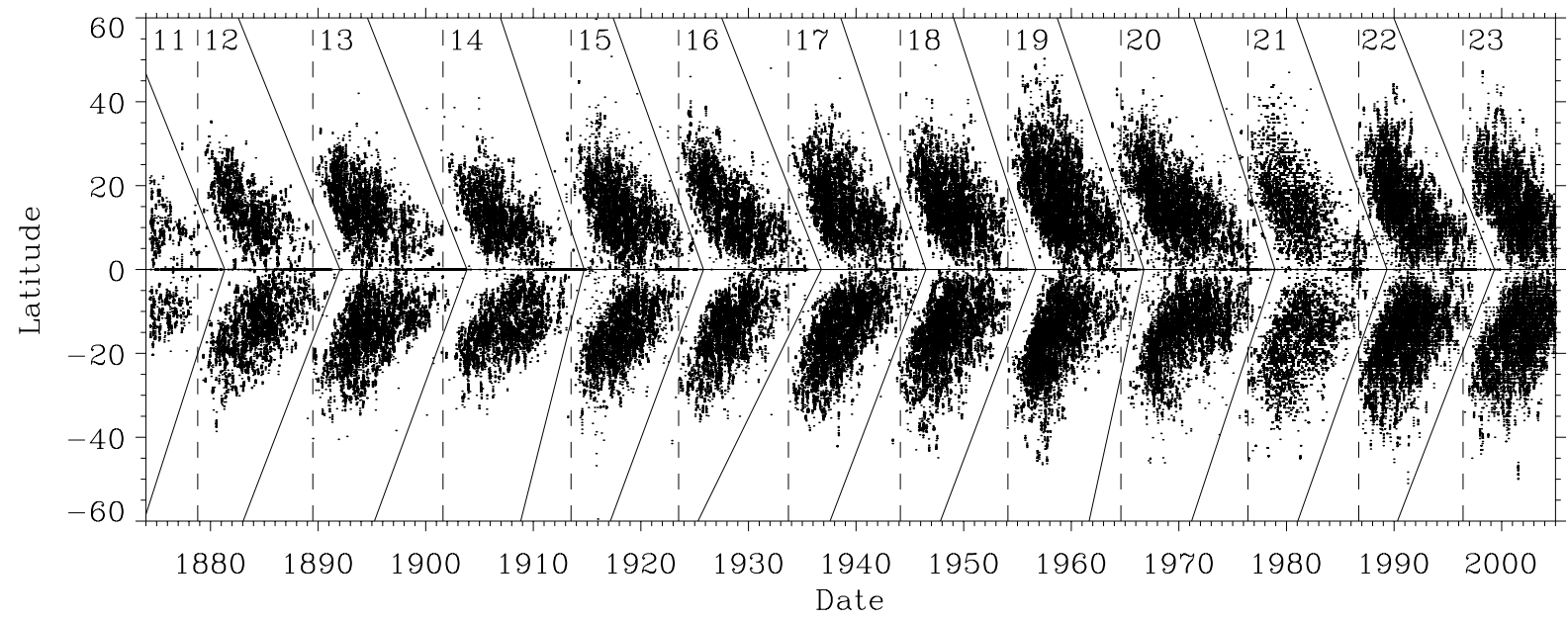

Fig. 1. Butterfly diagram from 1874 to 2005 based on data from the Royal Greenwich Observatory up to 1976, from the former Soviet Union between 1977 and 1985 and from the Mount Wilson Solar Observatory from 1986 onward. The vertical dashed lines mark the official minima of the sunspot cycle, the inclined solid lines our estimates of the boundaries between individual cycles. Cycle numbers are marked at the top of each cycle.

(Ossendrijver et al. 1996), the interplay between the dynamo at the base of the convection zone and magnetic fields inside it (Schmitt et al. 1996), and, finally, a variation of the meridional circulation (Charbonneau \& Dikpati 2000).

Some preliminary results, based on a simpler analysis and a different data set, were presented by Solanki et al. (2000). A somewhat similar analysis was also published by Li et al. (2003), who, however, did not use moments and basically considered only peak sunspot areas, sunspot areas at high latitudes $\left(>35^{\circ}\right)$ and cycle mean latitudes.

The paper is structured as follows. In Sect. 2 we describe the solar data, in Sect. 3 the technique, while in Sect. 4 results obtained from the solar data are presented. In Sect. 5 we present some simple dynamo calculations which are analysed in the same way as the historical sunspot data. The results of the analysis of the models are given in Sect. 6. Finally, we outline our conclusions in Sect. 7.

\section{Sunspot data}

The analysed data are the sunspot positions and areas collected by the Royal Greenwich Observatory (RGO) between 1874 and 1976 and by other observatories since then. The exact data set used has been described by Balmaceda et al. (2005). For the period 1977-1985, after the end of the Greenwich observations up to the official minimum of cycle 21 , it is based on data from the former Soviet Union (called Russian data in the following) and for the period 1986-2004 on the Mount Wilson Solar Observatory record, which is composed of a collection of data from different sources. Data from Yunnan, Catania and Rome were used to fill gaps in the above-mentioned data sets wherever possible. One problem when combining, in particular, sunspot areas from different sources is that sunspot areas measured at different observatories often differ systematically (e.g. Fligge \& Solanki 1997; Foster 2004; Hathaway, priv. comm.; see http://science.nasa.gov/ssl/pad/solar/ sunspots.htm). Balmaceda et al. (2005, 2007) have therefore intercompared the various data sets and normalized all sunspot areas to the RGO record in order to form a homogenous data set.

\section{Technique}

In a first step of the analysis we found the boundary between consecutive cycles. We considered the latitudes between $4^{\circ}$ and $24^{\circ}$ where there are a sufficient number of sunspots. $2^{\circ}$ wide bins were made over this range. For each set of these latitude bins we found the boundary between two cycles by looking for the one year wide interval for which the number of sunspots in that bin was smallest. In numerous cases the minimum within a latitude bin was broad and flat. Then the boundary between cycles was estimated by eye near the middle of this interval. Finally, a straight line was fit to the minimum times determined for a hemisphere. This line was subsequently employed as the boundary between cycles. In Fig. 1 we plot the sunspot butterfly diagram for the analysed period. The identification of cycles and of the boundaries between them (the inclined straight lines) are indicated. The data from the incompletely covered cycles 11 and 23 were not analysed, but were used to determine the boundaries of cycles 12 and 22, respectively. The results of the subsequent analysis turned out to be unaffected by the exact positions of these boundaries.

In a second step, once each sunspot group had been assigned to a given solar cycle, we added together (over time) the areas of all the sunspots belonging to that cycle and lying within a given latitude bin (bin width $0.1^{\circ}$ ). In this way we obtained for each cycle a two-lobed profile as a function of latitude, with one lobe each on the southern and the northern hemisphere of the Sun. The resulting latitude profiles of cycles Nos. 12 and 19, the weakest and strongest of the analysed cycles, respectively, are illustrated in Fig. 2. These are the data which we analysed further. Note that in step 3 we considered each hemisphere individually and compute the 0 th to 4 th moments, $m_{0}$ to $m_{4}$, defined as follows:

$$
\begin{aligned}
& m_{0}=\int_{0}^{\left|l_{\max }\right|} A(l) \mathrm{d} l, \\
& m_{1}=\frac{1}{m_{0}} \int_{0}^{\left|l_{\max }\right|} A(l) l \mathrm{~d} l, \\
& m_{j}=\frac{1}{m_{0}} \int_{0}^{\left|l_{\max }\right|} A(l)\left(l-m_{1}\right)^{j} \mathrm{~d} l, \text { with } j=2,3,4 .
\end{aligned}
$$




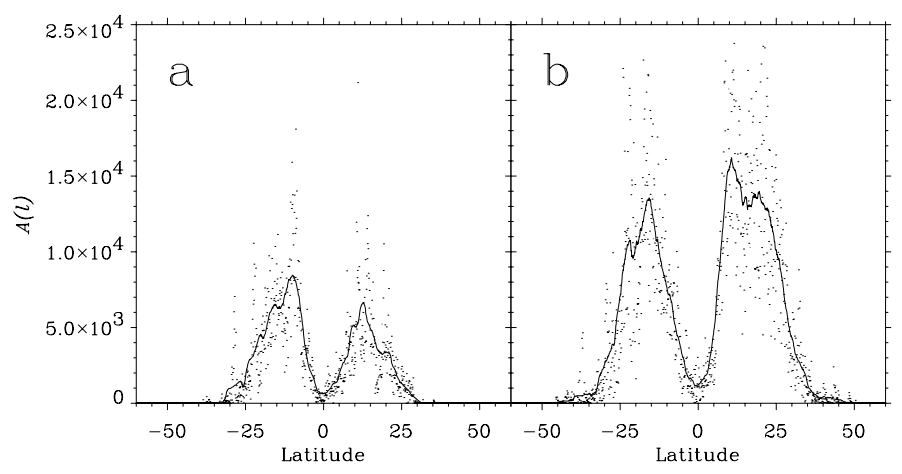

Fig. 2. Two examples of the latitude distribution of the cycle-integrated sunspot area. a) cycle 12, b) cycle 19 (dots). The solid lines represent $5^{\circ}$ running means.

Here $l$ is the latitude and $A(l)$ is the cycle-integrated sunspot area as a function of latitude. For $l_{\max }$ we have chosen $\pm 60^{\circ}$, since practically no sunspots have been known to occur at higher latitudes. The integration was carried out using a five-point NewtonCotes integration formula. A small error may be introduced by choosing the equator to be the boundary of the two wings of the butterfly, since a few spot groups are known (from their polarity signatures) to lie on the "wrong side of the equator". Spots within $2.5^{\circ}$ of the equator account for less than $1.5 \%$ of the total area covered by sunspots. Since spots of the opposite polarity form only a fraction of the spots close to the equator, we estimated a relatively small error produced by the use of the equator to demarcate the boundary between the hemispheres. Due to the lack of magnetic polarity data we could not improve on this in a simple manner. The moments are related to commonly used statistical parameters as follows:

$A_{\text {tot }}=m_{0}$,

$\langle l\rangle=m_{1}$,

$\sigma=\sqrt{m_{2}}$

$s=m_{3} / \sigma^{3}$,

$k=m_{4} / \sigma^{4}-3$.

Here $A_{\text {tot }}$ is the total solar surface area covered by sunspots over the solar cycle and $\langle l\rangle$ corresponds to the mean latitude at which sunspots are located during a given cycle. The moment $m_{2}$ represents the variance in latitude of the sunspot distribution, so that $\sigma$ is the standard deviation, a measure of the width of the latitude distribution of sunspots, $s$ is the skewness, a measure of the asymmetry in latitude of the cycle-integrated sunspot distribution. The skewness is zero for a symmetric profile and positive if the profile is skewed more towards the equator. The kurtosis $k$ is another parameter describing the shape of a distribution. As defined in Eq. (8) $k$ is zero for a Gaussian profile, positive for a sharper (more peaked) profile and negative for a more boxlike profile. It is important to consider $s$ and $k$ instead of $m_{3}$ and $m_{4}$, since these higher order moments are intrinsically correlated with $m_{2}$. Dividing by $\sigma^{3}$ and $\sigma^{4}$, respectively, removes this artificial correlation, making $\sigma, s$ and $k$ a priori independent of each other. In principle it is also possible to compute moments of still higher order, but they are not so directly related as $m_{0}-m_{4}$ to intuitively accessible quantities. In addition, the higher order moments give increasing weight to large $|l-\langle l\rangle|$ values, i.e. to high latitudes and the equator (see Eq. (3)). There are few sunspots at these latitudes and consequently the statistical uncertainties increase with the order of the moment.

Finally, we compared the different moments of a given cycle with each other. We did this individually for the two hemispheres, with the $i$ th moment relating to the northern/southern hemisphere being referred to as $m_{i}(N)$ and $m_{i}(S)$, respectively. We also formed the average of the two: $m_{i}=\frac{1}{2}\left(m_{i}(N)+m_{i}(S)\right)$. Similarly, $\langle l\rangle_{N}$ and $\langle l\rangle_{S}, \sigma_{N}$ and $\sigma_{S}$, etc. refer to the values obtained in the northern and southern hemisphere, respectively, of the parameters introduced in Eqs. (4)-(8).

When analysing the data there are two points that need to be considered particularly carefully. The first is data gaps. The sunspots present on the solar disk on days on which no measurements were carried out do not contribute to the statistics. Two measured parameters of each sunspot enter into our analysis, its latitude and its area. The error introduced into the mean latitude, width of the latitude distribution, etc. (i.e. to $m_{1}$ and higher moments) due to missing data is a second order effect. E.g. if the number of data gaps is not too large and reasonably evenly distributed over a cycle, we expect the effect to be almost negligible, since each integral over $A(l)$ is normalized by dividing by $m_{0}$. Hence data gaps increase the random errors in the $m_{1}-m_{4}$ values, but do not introduce systematic errors unless they are concentrated at the beginning or the end of a cycle. This does preclude the use of incompletely sampled cycles (where, e.g., the beginning or end is missing). The situation is very different for $m_{0}$, into which the actual numerical values of $A(l)$ enter without normalization. Any missing sunspot data leads to a corresponding decrease in $m_{0}$. In order to avoid such systematic errors it is necessary to interpolate the sunspot areas across data gaps (but not the sunspot latitudes). We have chosen to carry out a linear interpolation. The other moments $m_{1}-m_{4}$ are not affected by this treatment.

The other potential pitfall is produced when data from different sources are combined (Fligge \& Solanki 1997; Foster 2004; Balmaceda et al. 2007). This has been carefully taken into account by Balmaceda et al. $(2005,2007)$ when constructing the employed data set (see Sect. 2). Nevertheless, in order to avoid the remaining uncertainty arising from the combination of different data sets, we have also carried out the analysis for the Greenwich data alone. The results in this case are rather similar to those obtained from the complete data set (see Sect. 4).

\section{Results based on observational data}

In Fig. 3 we plot the various parameters defined by Eqs. (4)-(8) against each other, for the northern hemisphere (left), the southern hemisphere (middle) and the average over both (right). Each dot represents the values for a given solar cycle. To save space only a selection of all the possible plots of this type is shown (these are in general combinations showing high correlations; see below).

Figure 3 reveals the range of variation of the different parameters. Thus, between cycles 12 and 22 the total hemispheric sunspot area varied between $8.69 \times 10^{5}$ and $3.21 \times 10^{6}$, with an average value of $1.72 \times 10^{6}$. In contrast to this factor of 3.7 between the $A_{\text {tot }}$ of different cycles, $\langle l\rangle$ and $\sigma$ varied by less than a factor of 1.5. From Fig. 3 it is also clear that $\sigma<\langle l\rangle$, which implies that the $\mathrm{N}$ and $\mathrm{S}$ sunspot distributions are distinct, which is also visible from Fig. 2 (cf. Fig. 5). Interesting is that the skewness is almost always positive, i.e. the profiles are skewed such that they peak closer to the equator, with a tail towards higher latitudes (the only exception is the southern part of cycle 14). In contrast, $k>0$ and $k<0$ values are equally common, suggesting 

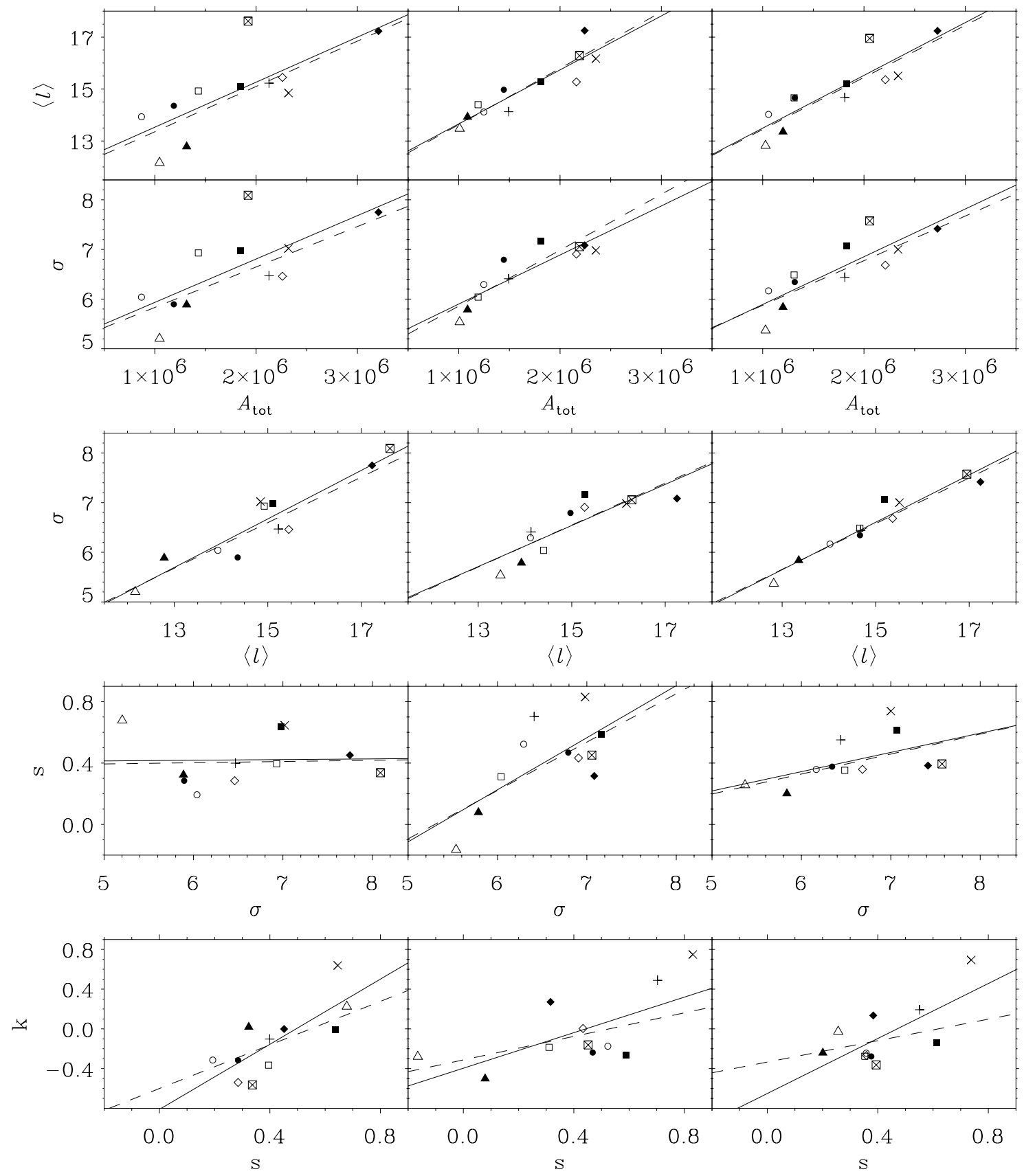

Fig. 3. Relationships between parameters describing the latitude dependence of the sunspot distribution. In the three columns we plot results obtained for the northern hemisphere (left), the southern hemisphere (middle) and the parameters averaged over both hemispheres (right). From top to bottom we plot $\langle l\rangle$ vs. $A_{\text {tot }}, \sigma$ vs. $A_{\text {tot }}, \sigma$ vs. $\langle l\rangle, s$ vs. $\sigma$ and $k$ vs. $s$. Open circles: cycle 12, filled circles: 13, open triangles: 14 , filled triangles: 15, open squares: 16, filled squares: 17, open diamonds: 18, filled diamonds: 19, pluses: 20, crosses: 21 , crossed squares: 22 . The solid lines are least squares fits. The dashed lines represent fits to cycles 12-20 alone (RGO data).

that compared to a Gaussian an almost equal number of cycles is box-shaped as is triangular. The values of these parameters averaged over all cycles are given in Table 1. The "uncertainties" listed in the table reflect the $1 \sigma$ scatter from one cycle to another.

Our main interest lies in the correlations between the different parameters, which can also be seen in Fig. 3. The linear correlation coefficient, $r_{\mathrm{c}}$, its uncertainty, $\delta_{+}$and $\delta_{-}{ }^{1}$ and the probability that this correlation is due to chance (false alarm probability), $P_{\mathrm{f}}$, are given in Table 2 , for all combinations of

\footnotetext{
${ }^{1}$ We refer to the $1 \sigma$ uncertainty in the correlation coefficients by $\delta$ in order to avoid confusion with the standard deviation of the cycleintegrated sunspot distribution introduced in Eq. (6).
}

parameters, obtained from the combined data set described in Sect. $2^{2}$. The comparisons between neighbouring moments are marked by an $\mathrm{X}$ in the last column. The three lowest moments are correlated at a remarkably high level. The false alarm probability is at or below $1 \%$ in all cases involving just $A_{\text {tot }},\langle l\rangle$ and $\sigma$. Hence, a cycle with a large amplitude has sunspots on average at a higher latitude and distributed over a wider range of latitudes. As $\langle l\rangle$ shifts poleward by $5^{\circ}, \sigma$ increases by $2^{\circ}$. This means that the area covered by sunspots at higher latitudes varies

\footnotetext{
2 A similar analysis using just Greenwich data, in order to avoid any uncertainty introduced by imperfect intercalibration between sunspot data sets, gives basically the same results.
} 
Table 1. Mean values of parameters of the solar cycles studied in this paper and their $1 \sigma$ cycle to cycle scatter.

\begin{tabular}{c|c|c|c}
\hline \hline Moment & $N$ & $S$ & $\langle N+S\rangle$ \\
\hline$A_{\text {tot }}$ & $(1.78 \pm 0.69) \times 10^{6}$ & $(1.66 \pm 0.51) \times 10^{6}$ & $(1.72 \pm 0.57) \times 10^{6}$ \\
$\langle l\rangle$ & $14.88 \pm 1.63$ & $15.03 \pm 1.17$ & $14.95 \pm 1.34$ \\
$\sigma$ & $6.61 \pm 0.85$ & $6.55 \pm 0.57$ & $6.58 \pm 0.66$ \\
$s$ & $0.42 \pm 0.16$ & $0.41 \pm 0.28$ & $0.42 \pm 0.16$ \\
$k$ & $-0.12 \pm 0.35$ & $-0.03 \pm 0.38$ & $-0.07 \pm 0.31$ \\
\hline
\end{tabular}

Table 2. Correlation coefficients, $1 \sigma$ uncertainties of the correlation coefficients and false alarm probability for the combined data set (1874-2004).

\begin{tabular}{|c|c|c|c|c|c|c|c|c|c|c|c|c|c|}
\hline & \multicolumn{4}{|c|}{$\overline{\bar{N}}$} & \multicolumn{4}{|c|}{$\overline{\bar{S}}$} & \multicolumn{4}{|c|}{$\overline{\langle\overline{\langle N+S\rangle}}$} & \\
\hline & $r_{\mathrm{c}}$ & $\delta_{-}$ & $\delta_{+}$ & $P_{\mathrm{f}}$ & $r_{\mathrm{c}}$ & $\delta_{-}$ & $\delta_{+}$ & $P_{\mathrm{f}}$ & $r_{\mathrm{c}}$ & $\delta_{-}$ & $\delta_{+}$ & $P_{\mathrm{f}}$ & \\
\hline$\langle l\rangle$ vs. $A_{\text {tot }}$ & 0.74 & 0.21 & 0.12 & 0.01 & 0.91 & 0.09 & 0.05 & $1 \times 10^{-4}$ & 0.87 & 0.12 & 0.06 & $5 \times 10^{-4}$ & $\mathrm{X}$ \\
\hline$\sigma$ vs. $A_{\text {tot }}$ & 0.71 & 0.22 & 0.14 & 0.01 & 0.88 & 0.11 & 0.06 & $3 \times 10^{-4}$ & 0.83 & 0.15 & 0.08 & $1 \times 10^{-3}$ & \\
\hline$\sigma$ vs. $\langle l\rangle$ & 0.93 & 0.07 & 0.03 & $3 \times 10^{-5}$ & 0.85 & 0.13 & 0.07 & $1 \times 10^{-3}$ & 0.97 & 0.03 & 0.02 & $1 \times 10^{-6}$ & $X$ \\
\hline$s$ vs. $A_{\mathrm{tot}}$ & 0.22 & 0.35 & 0.30 & 0.52 & 0.57 & 0.29 & 0.19 & 0.07 & 0.52 & 0.30 & 0.21 & 0.10 & \\
\hline$s$ vs. $\langle l\rangle$ & -0.15 & 0.32 & 0.35 & 0.65 & 0.41 & 0.33 & 0.25 & 0.21 & 0.39 & 0.33 & 0.25 & 0.24 & \\
\hline$s$ vs. $\sigma$ & 0.02 & 0.34 & 0.34 & 0.95 & 0.70 & 0.23 & 0.14 & 0.02 & 0.53 & 0.30 & 0.21 & 0.09 & X \\
\hline$k$ vs. $A_{\mathrm{tot}}$ & 0.16 & 0.35 & 0.31 & 0.64 & 0.60 & 0.27 & 0.18 & 0.05 & 0.47 & 0.31 & 0.23 & 0.14 & \\
\hline$k$ vs. $\langle l\rangle$ & -0.37 & 0.26 & 0.34 & 0.26 & 0.46 & 0.32 & 0.23 & 0.15 & 0.14 & 0.35 & 0.32 & 0.67 & \\
\hline$k$ vs. $\sigma$ & -0.17 & 0.31 & 0.35 & 0.61 & 0.42 & 0.33 & 0.24 & 0.20 & 0.16 & 0.35 & 0.31 & 0.64 & \\
\hline$k$ vs. $s$ & 0.76 & 0.19 & 0.11 & $6 \times 10^{-3}$ & 0.66 & 0.25 & 0.16 & 0.03 & 0.70 & 0.23 & 0.14 & 0.02 & $\mathrm{X}$ \\
\hline
\end{tabular}

much more strongly from one cycle to the next than the amplitude of the cycle as a whole, in agreement with the results of $\mathrm{Li}$ et al. (2003).

Correlations involving $k$ and $s$ are on the whole quite weak. Only a few individual correlations between $k$ and $s$ and between $s$ and $\sigma$ give a false alarm probability significantly below $10 \%$ in Table 2.

Another point that can be gleaned from Table 2 and from Fig. 3 is that the southern and northern hemispheres behave rather differently. Thus, the range covered by a given parameter differs, with $A_{\text {tot }},\langle l\rangle$ and $\sigma$ covering smaller ranges in the south, while for skewness the situation is reversed. Southern correlations are always positive (always $>0.4$ for the combined data set), while the northern hemisphere gives rise to weak (not significant) negative correlation coefficients involving $s$ and $k$. In general, the correlations are significantly stronger for the south. Only in 2 cases in Table 2 does the northern hemisphere exhibit a higher correlation, namely of $\sigma$ vs. $\langle l\rangle$ and for $k$ vs. $s$. This can also be seen from Fig. 4 in which differences between $r_{\mathrm{c}}$ values for $N$ and $S$ are plotted versus the $r_{\mathrm{c}}$ of the averaged moments (note that the symbols have a different meaning than in Fig. 3; see the figure caption). Obviously, the difference between high $r_{\mathrm{c}}$ values is small, while for low $r_{\mathrm{c}}$ values the difference is large.

Finally, there is a tendency for a stronger correlation between neighbouring moments. Thus, the correlation between $m_{0}$ and $m_{1}$, (i.e. between $\langle l\rangle$ and $A_{\text {tot }}$ ) and between $m_{1}$ and $m_{2}$ (i.e. between $\langle l\rangle$ and $\sigma$ ) is stronger than between $m_{0}$ and $m_{2}$ (or $A_{\text {tot }}$ and $\sigma)$. Similarly, $r_{\mathrm{c}}(s, \sigma)>r_{\mathrm{c}}(s,\langle l\rangle)>r_{\mathrm{c}}\left(s, A_{\mathrm{tot}}\right)$ (averaged over both hemispheres). The effect is weak, however, and not universal, since for the Greenwich data the kurtosis $k$ is best correlated with $m_{0}$, at least for the south and when averaged over both hemispheres. For the northern hemisphere $r_{\mathrm{c}}(k, s)$ is by far the highest. It should be recalled, however, that $k$ correlates poorly with the lower moments, with the probability of a false alarm reaching values as high as 0.67 (see Table 2).

It is difficult to determine how the latitudinal shape of the sunspot distribution over a solar cycle changes from cycle to cycle just from the data (e.g. Fig. 2) due to the scatter of the data points from one bin to another. This scatter can be reduced by choosing broader bins (see Li et al. 2003, who took $5^{\circ}$ wide bins). An alternative is to compute the running mean over the data points, as illustrated in Fig. 2.

In Fig. 5 we display the profiles smoothed by computing $5^{\circ}$ running means of each cycle. The profile shape varies considerably from one cycle to the next. As in the case of the temporal profiles of cycles, the latitudinal profiles show one, two or even more humps. Even at the level of smoothing introduced to make Fig. 5 the shape of the latitude profile still reflects sub-structure, which could be termed "active latitudes". It may well be related to the latitudes of higher activity seen in 6-month time-averaged sunspot area latitude distributions by Antalova \& Gnevyshev (1983). A more detailed analysis would be needed to ascertain the reality on any active latitudes, e.g. $13^{\circ} \mathrm{N}$, at which 5 cycles display a peak. In particular, it must still be ascertained how robust such peaks are to different amounts of averaging.

Figure 5 clearly shows that the low latitude part of the cycle remains relatively unchanged from one cycle to another (the curves representing the different cycles lie very close together), while the higher latitude parts of the cycle exhibit a considerable scatter. This agrees well with the result deduced from the moments: stronger cycles peak at higher latitudes and are broader, with a (weak) tendency to be more strongly skewed towards the equator, which also implies that the cycles will be more similar near the equator than at higher latitudes. Hence, the correlations we find between the moments suggest that changes at latitudes $\gtrsim 15^{\circ}\left(\lesssim-15^{\circ}\right)$ are well correlated with the bulk of the cycle. Also evident from Fig. 5 is the difference in the behaviour of the 2 hemispheres. The northern hemisphere exhibits a larger scatter in amplitude and width (cf. Fig. 3). There are also more cycles which show anomalous behaviour immediately to the north of the equator than to its south. Also plotted in Fig. 5 is the latitudinal profile averaged over all cycles (thick black curve). The $N$ and $S$ lobes of this profile are similar in strength, but differ, e.g., in asymmetry, with the profile in the south being more symmetric than that in the north. 

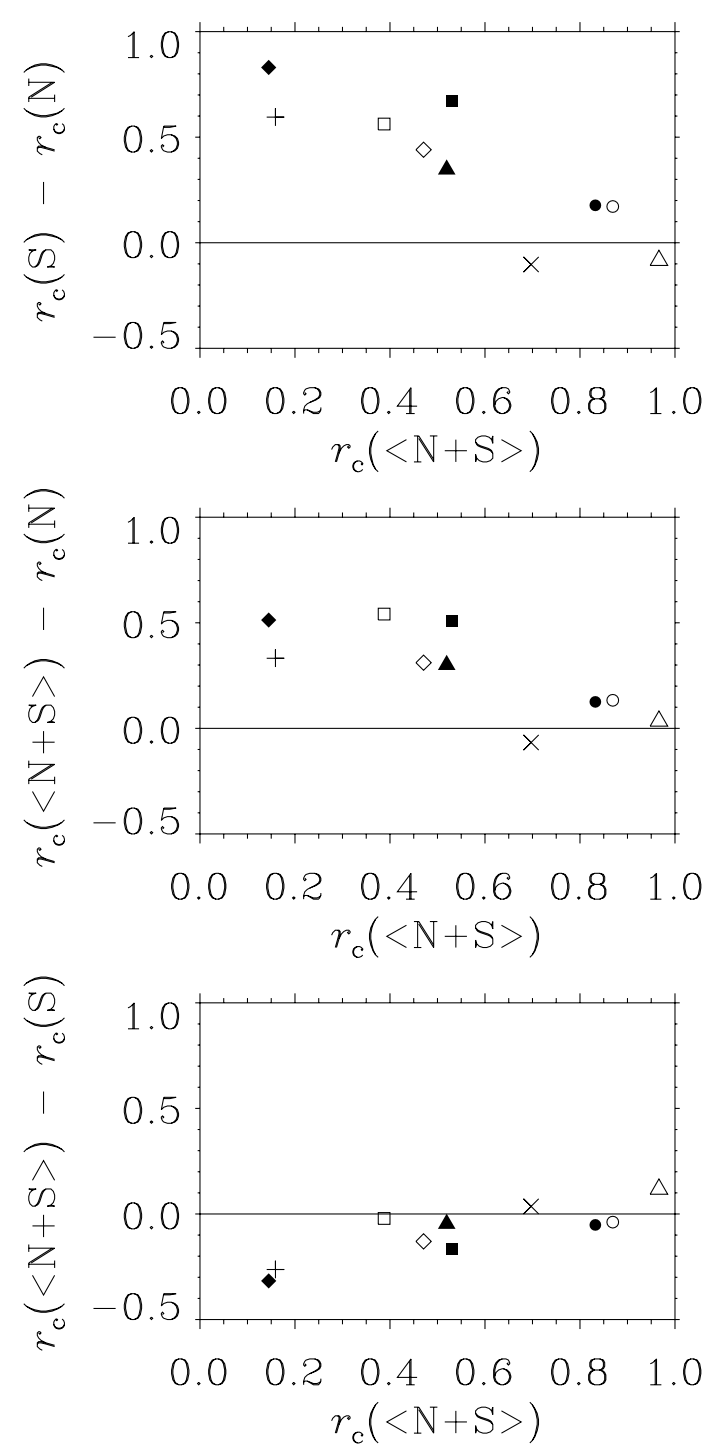

Fig. 4. Difference between correlation coefficients $r_{\mathrm{c}}$ between "moments" of latitude distributions of sunspots in the northern, $r_{\mathrm{c}}(N)$ and the southern hemispheres, $r_{\mathrm{c}}(S)$ vs. $r_{\mathrm{c}}(\langle N+S\rangle)$ (top panel). In the middle and bottom panels the difference between $r_{\mathrm{c}}(\langle N+S\rangle)$, and $r_{\mathrm{c}}(N)$ or $r_{\mathrm{c}}(S)$, respectively, are plotted. Open circles: $\langle l\rangle$ vs. $A_{\mathrm{tot}}$, filled circles: $\sigma$ vs. $A_{\text {tot }}$, open triangles: $s$ vs. $A_{\text {tot }}$, filled triangles: $k$ vs. $A_{\text {tot }}$, open squares: $\sigma$ vs. $\langle l\rangle$, filled squares: $s$ vs. $\langle l\rangle$, open diamonds: $k$ vs. $\langle l\rangle$, filled diamonds: $s$ vs. $\sigma$, pluses: $k$ vs. $\sigma$, crosses: $k$ vs. $s$.

\section{Dynamo models}

In order to test whether the correlations presented in Sect. 4 can be used as a new diagnostic of the solar dynamo, e.g., to distinguish between different dynamo models, we have carried out a set of computations with variants of a simple dynamo model. In order to simulate the irregularity of the solar cycle we allowed for stochastic fluctuations of the $\alpha$-effect (models A, B and C), a random source term due to magnetic fields from a turbulent convection zone dynamo (model D), and a variable meridional flow (model E).

We did not aim at a completely realistic and detailed model of the solar cycle and thus restricted ourselves to the thin shell dynamo of Schmitt \& Schüssler (1989) with prescribed radial dependence in the form of a spherical wave $\sim \exp (\mathrm{i} k r) / k r$ (Hoyng et al. 1994) such that the axisymmetric magnetic field, described by the toroidal components of the magnetic field, $B$, and the

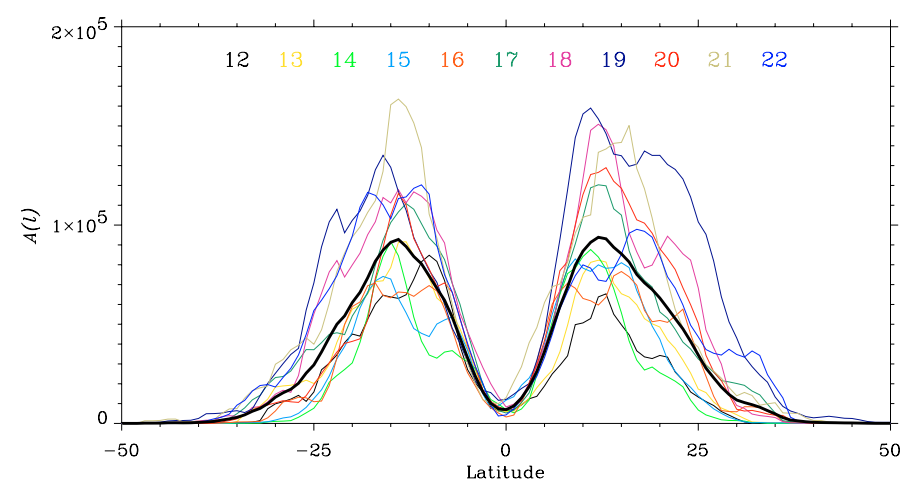

Fig. 5. Latitude distributions of the cycle-integrated sunspot area. $5^{\circ}$ running means are plotted. Colour key is given at the top of the plot. The thick black line represents the mean cycle distribution.

vector potential, $A$, only depends on the colatitude $\vartheta$ of the spherical shell and on time $t$. The dimensionless $\alpha \Omega$-dynamo equations are written as

$\frac{\partial B}{\partial t}=D(B)+R_{\Omega} \frac{\partial A \sin \vartheta}{\partial \vartheta}-R_{v} \frac{\partial v_{\vartheta} B}{\partial \vartheta}$
$\frac{\partial A}{\partial t}=D(A)+R_{\alpha} \alpha B+\delta S(\vartheta, t)-R_{v} \frac{v_{\vartheta}}{\sin \vartheta} \frac{\partial A \sin \vartheta}{\partial \theta}$

with diffusion operator

$D=\frac{1}{\sin \vartheta} \frac{\partial}{\partial \vartheta} \sin \vartheta \frac{\partial}{\partial \vartheta}-\frac{1}{\sin ^{2} \vartheta}-(k R)^{2}$,

Reynolds numbers of the $\alpha$-effect, differential rotation and meridional flow

$R_{\alpha}=\frac{\alpha_{m} R}{\eta}, \quad R_{\Omega}=\frac{\Omega^{\prime} R^{3}}{\eta}, \quad R_{v}=\frac{v_{m} R}{\eta}$

and a stochastic source term $\delta S(\vartheta, t)$. The $\alpha$-effect consists of a regular $\alpha$-effect and random fluctuations, $\alpha=\alpha_{0}(\vartheta, B)+$ $\delta \alpha(\vartheta, t) / \alpha_{\mathrm{m}}$, for the differential rotation we assumed a constant radial gradient $\mathrm{d} \Omega / \mathrm{d} r=\Omega^{\prime}$, and as part of the meridional circulation a flow in latitudinal direction $v_{\vartheta}=\sin 2 \vartheta+\delta v(\vartheta, t) / v_{\mathrm{m}}$, again with regular and fluctuating components. $\alpha_{\mathrm{m}}$ and $v_{\mathrm{m}}$ represent the maximum values of the regular parts of the $\alpha$-effect and the meridional flow, respectively. The length scale $R=$ $5 \times 10^{10} \mathrm{~cm}$ was chosen as the radial position of the dynamo layer at the bottom of the convection zone. We took the diffusion time, $R^{2} / \eta$, as the time scale, where $\eta$ is the turbulent magnetic diffusivity, and measured $B$ in units of $B_{0}=10^{5} \mathrm{G}$. Further we set $k R=3$. The dynamo equations were solved in the northern hemisphere with antisymmetric boundary condition for the magnetic field across the equatorial plane.

From the obtained magnetic fields only those above a threshold in field strength were further analysed. This accounts for the fact that only the stronger fields become buoyantly unstable, rise to the surface and contribute to sunspots. In models A, B and D we chose a threshold of 0.3 of the maximum value of the field strength reached during the whole run, in models $\mathrm{C}$ and $\mathrm{E}$ this threshold was set to 0.05 . The results do not significantly depend on the threshold.

In models $\mathrm{A}, \mathrm{B}$ and $\mathrm{C}$ we allowed for random fluctuations of the $\alpha$-effect superposed on the regular $\alpha$-effect in order to simulate the irregularity of the solar cycle. While in models $\mathrm{A}$ and $\mathrm{B}$ the regular $\alpha$-effect extends from equator to pole as $\alpha_{0}=\cos \vartheta$, it is confined to latitudes below $40^{\circ}$ in model $\mathrm{C}$ by taking $\alpha_{0}=\sin \left(\pi\left(\vartheta-\vartheta_{0}\right) /\left(\pi / 2-\vartheta_{0}\right)\right)$ for $\vartheta>\vartheta_{0}=\pi 50 / 180$ and 

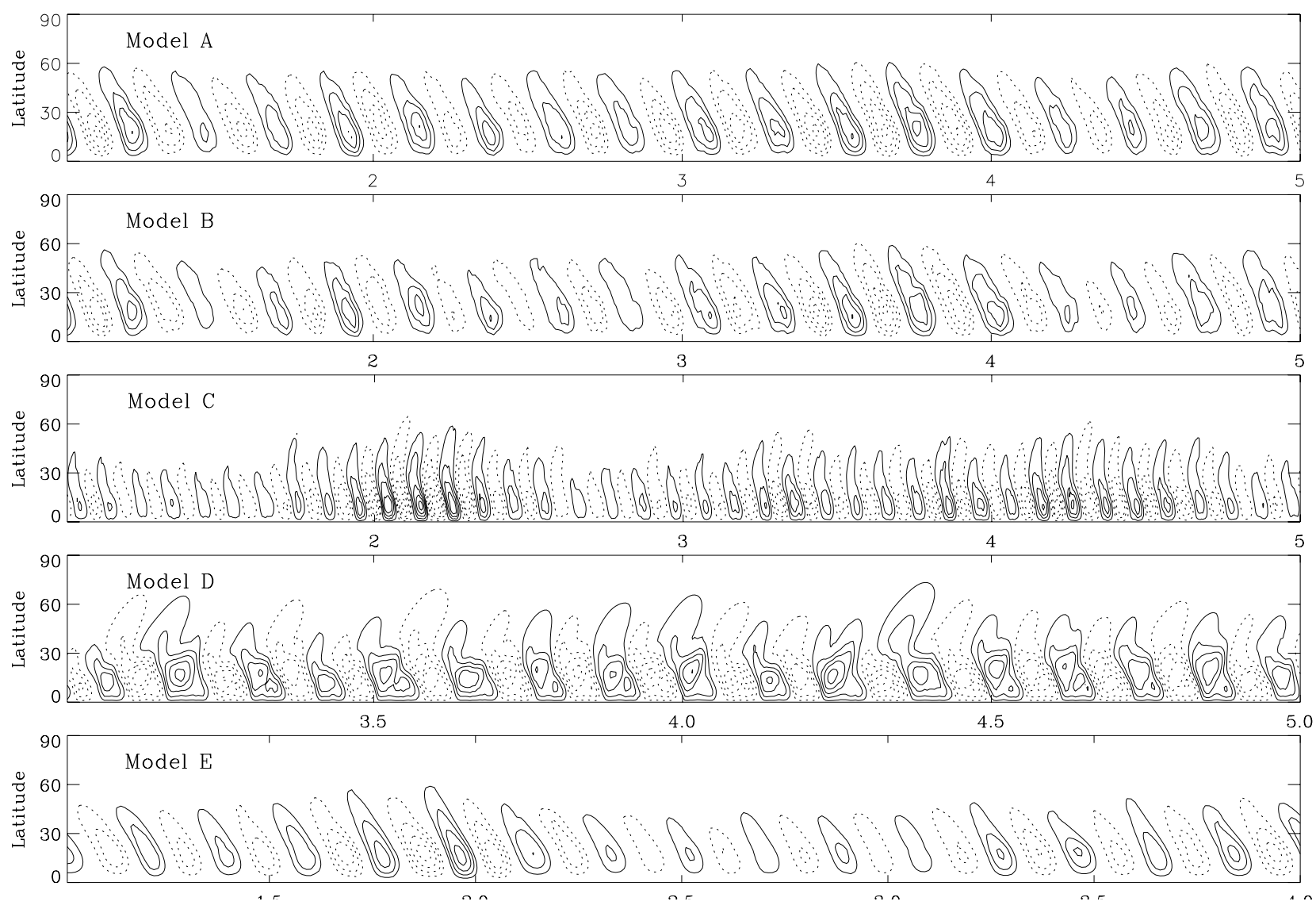

Fig. 6. Butterfly diagrams for the northern hemisphere produced by 5 simple dynamo models described in the text. Shown are contours of field strength, with negative fields being indicated by dashed contours. Time is measured in units of diffusion time.

$\alpha_{0}=0$ elsewhere, yielding butterfly wings closer to the equator in the latter case. These models were run at their corresponding critical excitation, i.e. $R_{\alpha} R_{\Omega}=-1300$ for models $\mathrm{A}$ and $\mathrm{B}$ and $R_{\alpha} R_{\Omega}=-2450$ for model C. The number of fluctuating cells from equator to pole was 50, which were renewed every 0.001 diffusion time steps, corresponding roughly to 1 month. The amplitudes $\delta \alpha / \alpha_{\mathrm{m}}$ of the fluctuations were random values from the interval $(-3,3)$, i.e. up to 3 times the regular $\alpha$-effect in models $\mathrm{A}$ and $\mathrm{C}$, and from the interval $(-4,4)$ in model B. 33 cycles were available for analysis of models $\mathrm{A}$ and $\mathrm{B}$, and 79 cycles for model $\mathrm{C}$. Note that only cycles produced after the models reached a statistically steady state were analysed. For details of the model setup and justification see Ossendrijver et al. (1996).

In model D we describe an overshoot layer dynamo with an $\alpha$-effect due to the buoyancy instability of the toroidal magnetic field (Ferriz-Mas et al. 1994). The regular $\alpha$-effect is limited to $40^{\circ}$ latitude as in model $\mathrm{C}$ and, additionally, operates only in a finite range of field strength, $B_{1} \leq B \leq B_{2}$, with $B_{1}=5 \times 10^{4} \mathrm{G}$ and $B_{2}=10^{5} \mathrm{G}$. Instead of $\alpha$-fluctuations a stochastic source term $\delta S(\vartheta, t)$ describes poloidal flux loops shed into the overshoot region from magnetic fields in the convection zone. The latitudinal extension, $l_{\mathrm{c}} \simeq 10^{5} \mathrm{~km}$, and the duration of such an injection, $t_{\mathrm{c}} \simeq 0.001$ diffusion times or 1 month, are determined by the length and time scales of the large-scale convective flows. We chose a random location $\vartheta_{0}$ and the amplitude of $\delta S$ is also a random number in the interval $\left(-\delta S_{\mathrm{m}}, \delta S_{\mathrm{m}}\right)$ with a dimensionless value of $\delta S_{\mathrm{m}}=0.3$. After each correlation time $t_{\mathrm{c}}$, new random values for $\vartheta_{0}$ and the amplitude of $\delta S$ are selected. The dynamo operated at twice the critical excitation, with $R_{\Omega}=-24500$ and
$R_{\alpha}=0.2$. Model details are given in Schmitt et al. (1996). The combination of a threshold in field strength for dynamo action and random fluctuations due to magnetic fields from a turbulent convection zone dynamo leads to activity cycles with amplitude variations, interrupted by randomly occurring grand minima. A time sequence without grand minima was chosen and a total of 33 cycles were available for analysis.

Finally in model $\mathrm{E}$ we investigated an equatorward flow as part of the meridional circulation at the location of the dynamo in the overshoot layer with superposed fluctuations (cf. Charbonneau \& Dikpati 2000). The flow speed was chosen to be rather low in order to keep the $\alpha \Omega$-dynamo mechanism from being quenched by the flow (see also Petrovay \& Kerekes 2004). In particular, for $R_{\Omega}=-1550, R_{\alpha}=1$ and $R_{v}=1$ the dynamo is critically excited, when a regular $\alpha$-effect with $\alpha_{0}=\cos \vartheta$ is adopted. The amplitudes of the fluctuations $\delta v(\vartheta, t) / v_{\mathrm{m}}$ are random numbers in the interval $(-2,2), 5$ cells from equator to pole were adopted, and renewed every 0.1 diffusion times, corresponding roughly to the duration of a cycle. 31 butterfly wings with amplitudes varying by a factor of about 3 are analysed.

In Fig. 6 we plot the analysed butterfly diagrams for each of the 5 models. The outermost contour in Fig. 6 corresponds to the relevant threshold level for each model. Solid and dashed contours refer to opposite polarity fields. Time is given in units of the diffusion time. Due to the applied boundary condition at the equator the southern hemisphere is just a mirror image multiplied by -1 . The difference between the butterfly diagrams resulting from the various models is clear already from this figure. 

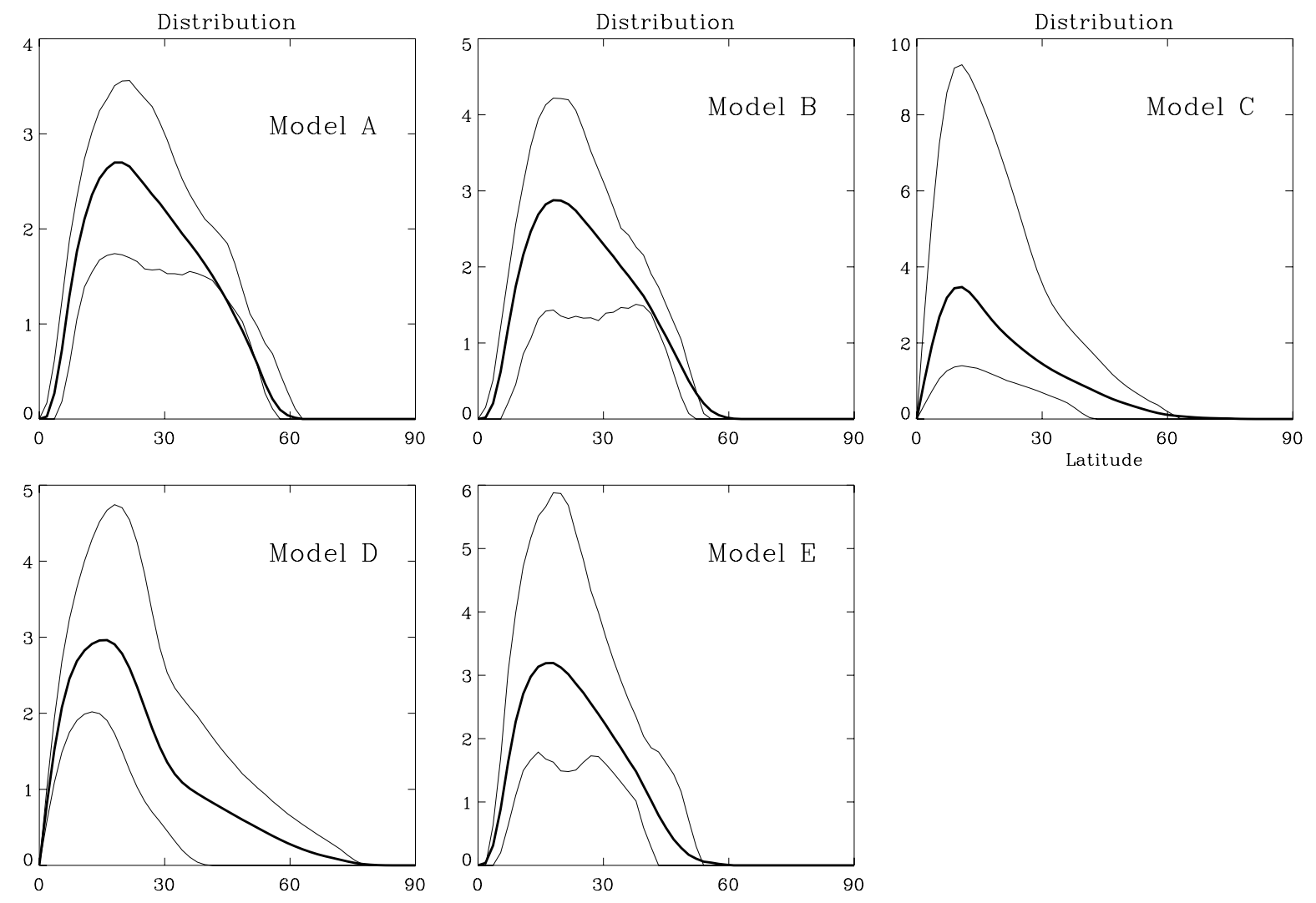

Fig. 7. Latitude distribution of cycle-averaged magnetic flux above the threshold for each of the 5 considered dynamo models. Plotted is the distribution averaged over all cycles (thick curves), as well as the distributions due to the strongest and weakest analysed cycles.

Models $\mathrm{C}$ and $\mathrm{E}$ show larger variability than the others, which is the reason that a different threshold was used for these models.

The synthetic butterfly diagrams were analysed in exactly the same manner as the solar data. The separation between individual cycles is straight forward for the models, since the sign of the toroidal field alternates between cycles. After integration over time we obtained latitudinal profiles, plotted in Fig. 7, similar to those obtained from the data. Shown are the average distribution of each model (i.e. averaged over all cycles; thick curves) as well as the distribution due to the weakest and the strongest cycle. Note that the weakest cycle has the lower $m_{0}$, but need not have the lowest magnetic flux at all latitudes. Similarly, the strongest cycle need not be the strongest at all latitudes.

\section{Results obtained with dynamo models}

Since the computed dynamos are symmetric around the equator we do not need to distinguish between the hemispheres as long as we do not directly compare with solar data. The results are summarised in Table 3.

There are similarities and differences between the models with regard to the correlations. Some correlation coefficients change comparatively little from one model to another, e.g., $\sigma$ vs. $\langle l\rangle$ or $k$ vs. $s$ while others are strongly dependent, such as $\langle l\rangle$ vs. $A_{\text {tot }}$ or $s$ vs. $\langle l\rangle$.

These very sensitive parameters, i.e. parameters which change significantly from one model to another, are of particular interest to distinguish between the models. Other such parameters include $s$ vs. $A_{\text {tot }}$ and $k$ vs. $A_{\text {tot }}$.

Model $\mathrm{E}$ is the only one for which all $r_{\mathrm{c}}$ values are positive. It has the highest $\left|r_{\mathrm{c}}\right|$ value in 6 out of 10 cases. There is also a tendency for model $\mathrm{C}$ to have more positive $r_{\mathrm{c}}$ values than models
A, B and D. The highest correlation is reached between $\sigma$ and $\langle l\rangle$, between $k$ and $s$, and to a lesser extent between $\sigma$ and $A_{\text {tot }}$. Whereas the first and third of these agrees well with the observations, $k$ and $s$, although reasonably related in the observational data $\left(r_{\mathrm{c}} \approx 0.66-0.76\right)$, is only the fourth highest correlation.

Comparing the $r_{\mathrm{c}}$ values in Table 3 with the values given in Table 2 shows some good agreement and some significant differences. Interestingly, the level of agreement depends strongly on whether a given model is compared with the northern or the southern solar hemisphere or with the full Sun.

To show these similarities and differences more clearly, we plot in Fig. 8 the difference between the solar and model correlation coefficients vs. the solar $r_{\mathrm{c}}$ value. It is interesting that models A-D (in particular C), i.e. models without a meridional flow, reproduce the behaviour of the northern solar hemisphere, which shows the weaker correlations between moments. Model E, on the other hand, lies closer to the behaviour of the whole Sun and the southern hemisphere. Therefore, the behaviour of the southern hemisphere of the Sun is better described by a model including a meridional circulation (in a simplified form).

In general, the probabilities that the correlations are due to chance are much smaller for the models, basically because of the larger number of cycles that could be calculated compared to the number for which observations are available.

\section{Discussion and conclusions}

We have analysed the 5 lowest moments of the latitude dependence of the solar cycle and have found tight relationships between the total strength of the sunspot cycle, the mean latitudes of the sunspots and the width of the latitude bands over which the sunspots are distributed. Correlations involving the skewness 
Table 3. Correlation coefficients, $1 \sigma$ uncertainties of the correlation coefficients and false alarm probability for dynamo models.

\begin{tabular}{|c|c|c|c|c|c|c|c|c|c|c|c|c|}
\hline & \multicolumn{4}{|c|}{ Model A } & \multicolumn{4}{|c|}{ Model B } & \multicolumn{4}{|c|}{ Model C } \\
\hline & $r_{\mathrm{c}}$ & $\delta_{-}$ & $\delta_{+}$ & $P_{\mathrm{f}}$ & $r_{\mathrm{c}}$ & $\delta_{-}$ & $\delta_{+}$ & $P_{\mathrm{f}}$ & $r_{\mathrm{c}}$ & $\delta_{-}$ & $\delta_{+}$ & $P_{\mathrm{f}}$ \\
\hline$\langle l\rangle$ vs. $A_{\mathrm{tot}}$ & 0.36 & 0.17 & 0.15 & 0.04 & 0.37 & 0.16 & 0.14 & 0.03 & 0.51 & 0.09 & 0.08 & $2 \times 10^{-6}$ \\
\hline$\sigma$ vs. $A_{\text {tot }}$ & 0.67 & 0.11 & 0.09 & $4 \times 10^{-4}$ & 0.78 & 0.08 & 0.06 & $4 \times 10^{-5}$ & 0.59 & 0.08 & 0.07 & $7 \times 10^{-8}$ \\
\hline$\sigma$ vs. $\langle l\rangle$ & 0.73 & 0.10 & 0.08 & $1 \times 10^{-4}$ & 0.73 & 0.09 & 0.07 & $1 \times 10^{-4}$ & 0.96 & 0.01 & 0.01 & $1 \times 10^{-14}$ \\
\hline$s$ vs. $A_{\text {tot }}$ & 0.27 & 0.18 & 0.16 & 0.12 & 0.44 & 0.16 & 0.13 & 0.01 & 0.42 & 0.10 & 0.09 & $7 \times 10^{-5}$ \\
\hline$s$ vs. $\langle l\rangle$ & -0.60 & 0.10 & 0.13 & $1 \times 10^{-3}$ & -0.39 & 0.14 & 0.16 & 0.03 & -0.02 & 0.11 & 0.12 & 0.85 \\
\hline$s$ vs. $\sigma$ & 0.002 & 0.18 & 0.18 & 0.99 & 0.24 & 0.18 & 0.16 & 0.16 & 0.24 & 0.11 & 0.11 & 0.02 \\
\hline$k$ vs. $A_{\text {tot }}$ & 0.14 & 0.18 & 0.17 & 0.39 & 0.17 & 0.18 & 0.17 & 0.32 & 0.32 & 0.11 & 0.10 & $2 \times 10^{-3}$ \\
\hline$k$ vs. $\langle l\rangle$ & -0.69 & 0.08 & 0.11 & $3 \times 10^{-4}$ & -0.58 & 0.11 & 0.13 & $1 \times 10^{-3}$ & -0.28 & 0.10 & 0.11 & $6 \times 10^{-3}$ \\
\hline$k$ vs. $\sigma$ & -0.34 & 0.15 & 0.17 & 0.05 & -0.21 & 0.16 & 0.18 & 0.21 & -0.08 & 0.11 & 0.12 & 0.41 \\
\hline \multirow[t]{3}{*}{$k$ vs. $s$} & 0.81 & 0.07 & 0.05 & $4 \times 10^{-5}$ & 0.74 & 0.09 & 0.07 & $8 \times 10^{-5}$ & 0.90 & 0.02 & 0.02 & $1 \times 10^{-13}$ \\
\hline & \multicolumn{4}{|c|}{ Model D } & \multicolumn{4}{|c|}{ Model E } & & & & \\
\hline & $r_{\mathrm{c}}$ & $\delta_{-}$ & $\delta_{+}$ & $P_{\mathrm{f}}$ & $r_{\mathrm{c}}$ & $\delta_{-}$ & $\delta_{+}$ & $P_{\mathrm{f}}$ & & & & \\
\hline$\langle l\rangle$ vs. $A_{\text {tot }}$ & 0.72 & 0.10 & 0.08 & $4 \times 10^{-6}$ & 0.65 & 0.12 & 0.10 & $8 \times 10^{-5}$ & & & & \\
\hline$\sigma$ vs. $A_{\text {tot }}$ & 0.62 & 0.13 & 0.10 & $4 \times 10^{-5}$ & 0.86 & 0.06 & 0.04 & $1 \times 10^{-6}$ & & & & \\
\hline$\sigma$ vs. $\langle l\rangle$ & 0.94 & 0.02 & 0.02 & $3 \times 10^{-8}$ & 0.85 & 0.06 & 0.04 & $1 \times 10^{-6}$ & & & & \\
\hline$s$ vs. $A_{\mathrm{tot}}$ & -0.11 & 0.18 & 0.19 & 0.40 & 0.71 & 0.11 & 0.08 & $2 \times 10^{-5}$ & & & & \\
\hline$s$ vs. $\langle l\rangle$ & -0.18 & 0.17 & 0.18 & 0.17 & 0.15 & 0.19 & 0.18 & 0.30 & & & & \\
\hline$s$ vs. $\sigma$ & 0.08 & 0.19 & 0.18 & 0.55 & 0.54 & 0.15 & 0.12 & $6 \times 10^{-4}$ & & & & \\
\hline$k$ vs. $A_{\text {tot }}$ & -0.23 & 0.17 & 0.18 & 0.09 & 0.64 & 0.13 & 0.10 & $9 \times 10^{-5}$ & & & & \\
\hline$k$ vs. $\langle l\rangle$ & -0.53 & 0.12 & 0.15 & $3 \times 10^{-4}$ & 0.04 & 0.19 & 0.19 & 0.78 & & & & \\
\hline$k$ vs. $\sigma$ & -0.39 & 0.15 & 0.17 & $5 \times 10^{-3}$ & 0.41 & 0.17 & 0.14 & $7 \times 10^{-3}$ & & & & \\
\hline$k$ vs. $s$ & 0.83 & 0.07 & 0.05 & $4 \times 10^{-7}$ & 0.96 & 0.02 & 0.01 & $2 \times 10^{-7}$ & & & & \\
\hline
\end{tabular}
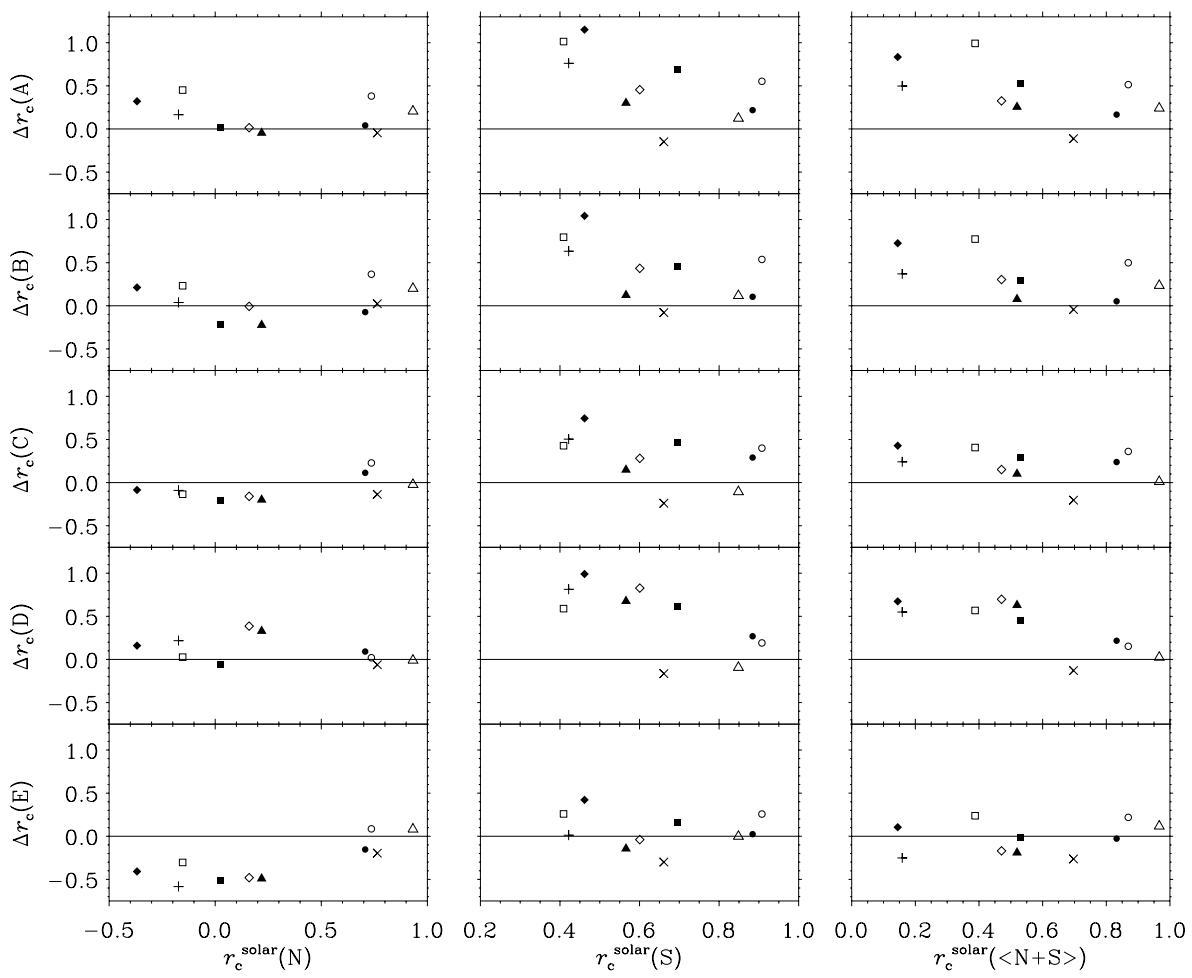

Fig. 8. Difference between correlation coefficients $r_{\mathrm{c}}$ obtained from solar data and from models, plotted vs. the observed $r_{\mathrm{c}}$. The three frames refer from left to right to solar $r_{\mathrm{c}}$ values deduced from the northern hemisphere, the southern hemisphere and the whole Sun. From top to bottom the frames refer to models A to E. Open circles: $\langle l\rangle$ vs. $A_{\mathrm{tot}}$, filled circles: $\sigma$ vs. $A_{\mathrm{tot}}$, open triangles: $s$ vs. $A_{\mathrm{tot}}$, filled triangles: $k$ vs. $A_{\mathrm{tot}}$, open squares: $\sigma$ vs. $\langle l\rangle$, filled squares: $s$ vs. $\langle l\rangle$, open diamonds: $k$ vs. $\langle l\rangle$, filled diamonds: $s$ vs. $\sigma$, pluses: $k$ vs. $\sigma$, crosses: $k$ vs. $s$.

and kurtosis of the latitude distribution of sunspots are weaker, although these two quantities do correlate significantly with each other. A clear difference between the hemispheres is evident: The correlations between all these parameters are stronger (and positive) for the southern hemisphere, while for the northern hemisphere some of the correlations are weakly negative, although at a level that is not significant. This type of analysis obviously brings out the differences between the hemispheres particularly clearly. 
Since sunspots are formed soon after the emergence of an active region and generally die long before the active region itself, they can be considered to be tracers of freshly emerged magnetic flux. Thus our results can be compared directly with models describing the generation of the Sun's magnetic field (dynamo models). We therefore tested the usefulness of the relations found here by computing butterfly diagrams for 5 simple dynamo models and analysing these in exactly the same way as the data. The aim here was not primarily to identify the most appropriate dynamo model, but rather to use these models to see if moments of cycle-averaged sunspot distributions have the power to distinguish between models. We found different levels of agreement with the real Sun from one model to the other. In particular, we could identify correlations between moments whose diagnostic value is high in that they could clearly distinguish between models.

The most striking (and surprising) result of our analysis concerns the north-south asymmetry of solar activity, which has been reported to manifest itself in various activity indices such as flares, filaments, magnetic flux and sunspot numbers and areas (e.g. Reid 1968; Howard 1974; Verma 1993; Temmer et al. 2002; Knaack et al. 2004, and references therein). The models without meridional flow (A-D) reproduce the latitude dependence of the sunspots in the northern solar hemisphere far better than in the southern hemisphere, while for the model with meridional flow (E) it is the other way round. This is as yet no proof that the meridional flow plays different roles in the two solar hemispheres, but it is intriguing and calls for more work. In particular, an analysis that goes beyond our exploratory work and involves the comparison with more realistic dynamo models is needed. In any case our result shows that the north-south asymmetry of solar activity manifests itself also in rather complex ways.

\section{References}

Antalova, A., \& Gnevyshev, M. N. 1983, Contributions of the Astronomical Observatory Skalnate Pleso, 11, 63
Balmaceda, L., Solanki, S. K., \& Krivova, N. A. 2005, Mem. Soc. Astron. It., 76,929

Balmaceda, L., Solanki, S. K., Krivova, N. A., \& Foster, S. 2007, J. Geophys. Res., submitted

Charbonneau, P. 2005, Living Rev. Sol. Phys., 2, 2

Charbonneau, P., \& Dikpati, M. 2000, ApJ, 543, 1027

Ferriz-Mas, A., \& Núñez, E. 2003, Advances in Nonlinear Dynamos (London and New York: Taylor \& Francis)

Ferriz-Mas, A., Schmitt, D., \& Schüssler, M. 1994, A\&A, 289, 949

Fligge, M., \& Solanki, S. K. 1997, Sol. Phys., 173, 427

Foster, S. S. 2004, Ph. D. Thesis, University of Southampton

Gnevyshev, M. N., \& Ohl, A. I. 1948, Astron. Zh., 25, 18

Harvey, K. L. 1992, The solar cycle, ASP Conf. Ser., 27, 580

Hathaway, D. H., Wilson, R. M., \& Reichmann, E. J. 1994, Sol. Phys., 151, 177

Hathaway, D. H., Wilson, R. M., \& Reichmann, E. J. 2002, Sol. Phys., 211, 357

Howard, R. 1974, Sol. Phys., 38, 59

Hoyng, P., Schmitt, D., \& Teuben, L. J. W. 1994, A\&A, 289, 265

Hoyt, D. V., \& Schatten, K. H. 1998, Sol. Phys., 181, 491

Knaack, R., Stenflo, J. O., \& Berdyugina, S. V. 2004, A\&A, 418, L17

Li, K. J., Wang, J. X., Zhan, L. S., et al. 2003, Sol. Phys., 215, 99

Mursula, K., Usoskin, I. G., \& Kovaltsov, G. A. 2001, Sol. Phys., 198, 51

Ossendrijver, A. J. H., Hoyng, P., \& Schmitt, D. 1996, A\&A, 313, 938

Ossendrijver, M. 2003, A\&AR, 11, 287

Pelt, J., Brooke, J., Pulkkinen, P. J., \& Tuominen, I. 2000, A\&A, 362, 1143

Petrovay, K., \& Kerekes, A. 2004, MNRAS, 351, L59

Reid, J. H. 1968, Sol. Phys., 5, 207

Schmitt, D., \& Schüssler, M. 1989, A\&A, 223, 343

Schmitt, D., Schüssler, M., \& Ferriz-Mas, A. 1996, A\&A, 311, L1

Solanki, S. K., \& Schmidt, H. U. 1993, A\&A, 267, 287

Solanki, S. K., Fligge, M., Pulkkinen, P., \& Hoyng, P. 2000, JA\&A, 21, 163

Solanki, S. K., Krivova, N. A., Schüssler, M., \& Fligge, M. 2002, A\&A, 396, 1029

Temmer, M., Veronig, A., \& Hanslmeier, A. 2002, A\&A, 390, 707

Usoskin, I. G., \& Mursula, K. 2003, Sol. Phys., 218, 319

Verma, V. K. 1993, ApJ, 403, 797

Waldmeier, M. 1935, Astron. Mitt. Zürich, 14, 105

Waldmeier, M. 1961, The sunspot-activity in the years 1610-1960 (Zurich: Schulthess), 171

Wilson, A. 2002, From Solar Min to Max: Half a Solar Cycle with SoHO, ESA SP-508

Wilson, R. M. 1988, Sol. Phys., 117, 269

Wolf, R. 1852, Acad. Sci. Compt. Rend., 35, 704 